\title{
REVISIONES
}

\section{Gestión de sí y educación del cuerpo en contexto: perspectivas y tensiones de la educación Argentina}

\author{
Construction and education of the body in context: \\ perspectives and tensions of education in Argentina
}

\author{
Gestão de si e educação do corpo em contexto: \\ perspectivas e tensões da educação Argentina
}

\section{María Valeria Emiliozzi}

CICES-IdHCS-CONICET-UNLP, 0221-155927127,val_emiliozzi@ hotmail.com

\section{RESUMEN}

El artículo da cuenta de los modos de constitución del cuerpo en la educación en general y los efectos de la Educación Física de la Provincia de Buenos Aires (Argentina) en particular, a partir de discursos del presente que irrumpen con la universalidad y la homogeneidad de la Educación y colocan la diferencia y el reconocimiento individual como punto de partida.

El trabajo identifica un desplazamiento que se produce en el curriculum, que lleva a poner en tensión al sujeto universal como objetivo y contenido de la educación, produciendo una apertura hacia el sujeto singular -el individuo- como punto de partida de la Educación.

Palabras claves: Curriculum, educación física, sujeto.

\section{ABSTRACT}

THE article reports on the types of construction of the body on education in general, and the particular case of the effects of Physical Education in the Province of Buenos Aires (Argentina) from the current discourse that appears with the universality and homogeneity of Education placing the difference and recognition of the individual as a starting point. The paper identifies a shift occurring in the curriculum, which enhances a tension point of the universal subject as the main purpose and content of education, this leads to the possibility of the singular subject -the individual -as the starting point of education.

Keywords: curricula, physical education, SUBJECT.

\section{RESUMO}

O artigo dá conta dos modos de constituição do corpo na educação em geral e dos efeitos da Educação Física da Província de Buenos Aires (Argentina) em especial, a partir de discursos do presente que emergem com a universalidade e a homogeneidade da Educação e colocam a diferença e reconhecimento individual como ponto de partida. Identifica-se um deslocamento produzido no currículo, pondo em tensão o sujeito universal como objetivo e conteúdo da educação, produzindo uma abertura para o sujeito singular - o indivíduo - como o ponto de partida da educação.

Palavras-chave: Currículo. Educação Física. Sujeito. 


\section{INTRODUCCIÓN}

El artículo surge a partir de un conjunto de interrogantes y reflexiones sobre el sujeto de la Educación, problema que retorna cada vez que se constituye una nueva reforma educativa y se establecen las reglas del sentido en las que se define una manera de pensarlo. El sistema educativo de la provincia de Buenos Aires (Argentina) no sólo ha colocado redes de sentido a partir de las cuales construir positivamente una manera de pensar la educación y su sujeto, sino que también ha provocado rupturas y acontecimientos que exigen repensar este sujeto y los fines mismos de la educación.

Los programas, los planes de estudio y el curriculum de la Educación Secundaria ${ }^{1}$ han sido una de las cuestiones más debatidas, no sólo en términos académicos sino también políticos, en la medida en que cada sociedad ha dado una respuesta a lo que considera que la escuela debe enseñar a los jóvenes. Como establece Dussel, "la legitimidad de nuestro accionar tiene que ver con la capacidad de dar cauce, ampliar o nutrir lo que la sociedad considera que es importante y relevante de enseñar" (Dussel, 2010: 4). Esto implica que el curriculum se constituye en un documento público, que posee una serie de reglas que marcan los sentidos de la educación y de la enseñanza: qué se enseña, cómo se enseña, y dentro de qué reglas se enseña. El documento es constituido por una serie de discursos que en el contexto de una positividad han podido aparecer, construyendo determinados sentidos a la educación y al cuerpo. Mariano Palamidessi plantea que:

La normativa curricular [...] es una de las instancias donde se define buena parte de las prevenciones, énfasis y atenciones que se dan cita en la cotidianidad de la escuela [...] Al formalizar los contenidos, recomendaciones metodológicas, fundamentaciones, bibliografías, el currículum organiza el campo posible de acción de maestros y alumnos, delimitando los objetos con los que debe interactuar en su experiencia (2000: 216).

En este sentido, el documento no queda encapsulado en las letras de la normativa, sino en un sistema de acción, en "el proceso de construcción curricular en el cual las instituciones educativas y los docentes configuran su acción educativa" (Bordoli, 2013: 189). Esto implica que, sea cual fuere su forma (documento, discurso, diálogo, preceptos), éste tiene por objeto principal la propuesta de reglas que establecen el hacer, el decir y el pensar de las prácticas.

En la actualidad, el sistema educativo establece un cambio en la manera de pensar la educación del cuerpo, y, a su vez, una reafirmación de ciertos principios y conceptos. En el caso específico de la Provincia de Buenos Aires (Argentina), en el año 2004 comienza a esbozarse una nueva reforma educativa que se construye en el marco de acontecimientos disruptivos que instalan la necesidad de introducir cambios en la estructura y organización del sistema educativo bonaerense, y crean las condiciones de posibilidad para que la Dirección General de Cultura y Educación elabore "una nueva propuesta pedagógica para la educación de los jóvenes adolescentes bonaerenses" (DGCyE, 2006: 10). La propuesta se plasmó en una nueva organización de la Educación Secundaria, que se constituyó, a partir del ciclo lectivo 2007, con 6 años de escolaridad distribuidos en 3 años de Educación Secundaria

Según el contexto histórico se han denominado planes y programas, o curriculum. Primeramente se llamaron planes o programas, que se ajustaban a conocimientos a transmitir. Luego, a partir de los ' 60 y '70, se denominaron curriculum, palabra que comprende el documento como práctica histórica, política y social que se da en el devenir del dispositivo pedagógico. 
Básica y 3 años de Educación Polimodal; y a partir del 2008, con 6 años de escolaridad distribuidos en 3 años de Educación Secundaria Básica y 3 años de Secundaria Superior. ${ }^{2}$

Ahora bien, ¿qué cambió?, ¿qué sentidos de la educación se pretendieron disipar?, ¿hay un modo diferente de pensar la educación o se persiste en las mismas ideas?, ¿qué saberes permiten pensar al sujeto de la educación?, o, ¿quién es el sujeto de la educación?

\section{EL HAZ DE RELACIONES DEL SISTEMA EDUCATIVO Y LOS NUEVOS ENFOQUES}

El dispositivo escolar se encuentra en el haz de relaciones de la sociedad industrial y forma parte del entramado institucional propio del desarrollo capitalista: la fábrica, la familia, la escuela, la clase social. Se trata de un sistema de pensamiento cuya eficacia resulta de la complementariedad de su acción y su común referencia al Estado Nacional. Por ello, la pedagogía procura recurrentemente:

ser la dueña del futuro y la constructora del mundo. Porque para fabricar el futuro y construir el mundo la pedagogía tenía que dominar a los niños que incorporaban el futuro por venir.

El acto de educar, en un modo institucional moderno, pone en funcionamiento una serie de fuerzas para dirigir, imponer e incluso dominar las fuerzas de los otros. En otras palabras, produce una sobrecodificación, una regulación, "una acción de una voluntad sobre otras definidas como jóvenes que no pueden solos con su voluntad (...) y por ello deben ser dirigidos (Grinberg y Levy, 2009: 24).

Por un lado, en una primera instancia, este progreso estuvo enfocado hacia la homogenización de la población, inicialmente hacia la normalización física y luego hacia la formación ciudadana, la cual se ha constituido en el sentido dominante del dispositivo escolar de fines del siglo XIX y principios del XX (Cf. Tedesco 1993). Por otro lado, la formación de recursos humanos ha sido el sentido dominante de la educación en el marco del desarrollo industrial que toma auge en el peronismo, además de ser un sentido que se impone vigorosamente en los ' 70 . El discurso pedagógico se encuentra convocado por el orden y el progreso, pues, de algún modo, el germen de la "fantasía didáctica" (Behares, 2004: 27) se encuentra en la escatología positivista que encuentra en la aplicación de los conocimientos el camino para el progreso de la humanidad.

Más allá de los diferentes saberes y condiciones de posibilidad que van formando y transformando la práctica educativa, ésta ha continuado operando en favor de cierta homogeneidad. Sin embargo, la crisis del estado del bienestar, la acumulación fordista, la desinversión, la desindustrialización, llegan hasta la reforma de los '90, produciendo un acontecimiento que pretende irrumpir con la homogenización que traía aires de progreso. El proceso de globalización establece nuevas relaciones de poder que deshacen el entramado institucional en el que se sostenía el dispositivo escolar, y pone en despliegue un proceso de desinstitucionalización que acompaña la crisis de la sociedad como concepto y realidad (Cf. Tenti Fanfani, 2002).

Si bien durante el año 2008 se produjeron cambios en la organización de los últimos tres años de escolaridad, los diseños curriculares de $1^{\circ}, 2^{\circ}$ y $3^{\circ}$ año no se modificaron. 
La desigualdad es el acontecimiento que opera y pone sobre la mesa algo no pensado. El efecto reproductivo de un sentido único, de una formación escolar en términos de una configuración de una identidad homogénea, encubre otras formas de cultura y produce desigualdad. La cuestión radica en que, si somos diferentes, el ser, la libertad de la elección de la identidad, la búsqueda de sí mismo, del yo interior, etc., deben ser los puntos a tomarse como referentes. Sin embargo, esto no implica que el Estado desaparezca, sino que se trata de nuevas prácticas educativas que se reestructuran en un nuevo tipo de relación entre el Estado y las escuelas. Esta restructuración, que se mantiene en el presente, no implica la ausencia del dispositivo pedagógico, sino su transformación en un nuevo dispositivo que produce un desplazamiento de las formas de gobierno (dirección de las conductas en relación a un deber ser) hacia las formas del auto-gobierno (dirección sobre sí mismo), que pone tanto al alumno como al maestro ante una nueva relación.

El horizonte de sentido que produce esta transformación entra en conexión con un exterior mediante la reordenación de conceptos. Se trata de un discurso que coloca al individuo como responsable de sí mismo, y dirige la práctica pedagógica hacia la formación, impulso y administración del yo. No se trata de suponer un sujeto, sino al revés: no se supone ningún sujeto, si no es autónomo es culpa suya. El individualismo moderno entiende al sujeto como interindividual, llevando a que el sujeto se haga responsable de sí mismo. En este contexto, la episteme no sostendrá el gobierno y la administración de los cuerpos, sino la gestión: "la gestión como nueva episteme" (Grinberg, 2006: 70). Si el gobierno refería a la disposición de los medios para conseguir un fin, la gestión se incorpora a los medios como objeto de la planificación-previsión, trabaja sobre el proceso, recoloca los objetos de la planificación y de la dirección de las conductas de los individuos (Cf. Grinberg, 2006). Gestionar no sólo implica disponer los medios, sino también crearlos y articularlos. En este sentido, el curriculum pretende:

llevar adelante una gestión participativa de la clase, que incluya a los jóvenes en la elaboración de las propuestas, propiciando la construcción de sistemas de representatividad en la toma de decisiones. Se espera que los estudiantes accedan a la información que brinda sustento a su hacer corporal y motor, tengan espacio para aportar su opinión y participen de la toma de decisión en aquellos asuntos que afectan sus procesos de aprendizaje (DGCyE, 2010: 32).

Cada efecto que se pone en circulación entra en resonancia o en contradicción, y exige un reajuste. Hoy cada quien debe elegir su camino, el trabajador se transforma en un buscador de trabajo y el ciudadano en un individuo responsable de sí. Estas condiciones forman reglas, normas y una manera de pensar al cuerpo de la educación que no debe ser entendida como un dato en la cronología de la educación, sino como una fuerza operante en la historia, un campo de corrientes históricas bipolares (Cf. Agamben, 2009: 151). Estos caracteres se desplazan entre la emergencia y el devenir, ya que la mirada está dirigida hacia la promoción de la diversidad como valor de identidad, quedando la homogenización en el lugar de la crítica. A la vez, la fragmentación que en su momento fue puesta en debate por el Estado no es una fuerza negativa, sino la apuesta para la formación de la subjetividad. En este sentido, el curriculum enuncia:

[...] formar un sujeto que pueda reconocer sus potencialidades y dificultades y las de su contexto, para poner en práctica procedimientos de mejora; del mismo modo que favorece la 
constitución del estudiante como ser autónomo, comprometido con las problemáticas sociales. El espíritu de colaboración, de convivencia en medios no habituales; la asunción de roles y la actitud responsable sobre aquello que la tarea asumida implica (DGCyE, 2011: 11).

Ante la diversidad, la producción del sujeto toma el camino hacia la búsqueda del Yo, del sí mismo, de la autonomía, y la función del maestro sufre una redefinición. Debe "prever los métodos y estilos de enseñanza que le permitan al alumno tomar conciencia del propio cuerpo, de la funcionalidades y potencialidades motrices del mismo" (DGCyE, 2003: 64). En otras palabras, se conforman unas relaciones de enseñanza centradas, no ya hacia el deber ser (saludable, ciudadano, trabajador), sino hacia el alumno, a su característica individual o propia. La práctica "involucra a la autorreflexión, a la autoconducción, al autodesarrollo" (Grinberg y Levy, 2009: 111). Si no hay un único modelo válido de ser ciudadano, de ser saludable, la pregunta por quién soy se transforma en el objetivo. El curriculum se enfoca hacia ello:

la propuesta de tareas grupales que posibiliten la construcción de proyectos compartidos tiene posibilidades de concreción en este espacio, en el cual los jóvenes pueden alcanzar mayor conocimiento de sí mismos y de los grupos que integran desde una posición crítica y constructiva (DGCyE, 2010: 28).

Por ejemplo en relación a la educación del cuerpo, estas formas de proyectar el acto educativo "son algunos ejemplos de intervención pedagógica que inciden en la asunción de posiciones críticas respecto de imágenes externas y modelizadas del cuerpo, la autoconciencia y estima de sí mismo (DGCyE, 2010: 29).

Estas relaciones van colocando al alumno como centro del proceso y punto de partida, dejando atrás la relación didáctica que parte del docente, en la medida en que aparece un nuevo espacio de normatividad y de regulación que llama al yo a fabricarse a sí mismo, a producir su propio mundo. Ya no se trata de una educación para la homogeneización porque produce desigualdad y acrecienta las diferencias: entonces, se parte del supuesto de que somos diferentes. La nueva norma es la diversidad, la búsqueda de la identidad, la construcción del propio proyecto; conocerse y reconocerse en la propia identidad.

La ciudadanía se ejerce desde las prácticas particulares de grupos y sujetos sociales. Estas prácticas ciudadanas son entonces prácticas que ponen al descubierto la trama de las relaciones sociales y por lo tanto la conflictividad de las interacciones. Desde la perspectiva que se adopta en este Diseño Curricular, la noción de interculturalidad se entrelaza con la concepción de ciudadanía para enfrentar los desafíos que implica educar en un contexto de diversidad cultural, diferencia social y económica, y actuar en el terreno de las relaciones sociales entendidas como producto del conflicto y no de la pasividad de la convivencia de los distintos grupos sociales y culturales.

La interculturalidad es, como señala Canadell, ante todo, una actitud, una manera de percibirse uno mismo y la propia cultura como partes integrantes de un complejo interrelacionado que llamamos mundo. Toda cultura se fundamenta en una manera de estar en el mundo y de percibirlo. [...] Por ello, una cultura no es solamente una manera particular de entenderla, sino una realidad propia (DGCyE, 2006: 14)

En este sentido, la identidad ciudadana, que en un primer momento se pretendió universalizar a través de la homogenización de la población para afianzar la constitución 
del Estado-nación, posee un doble vínculo. Si bien, continúa la formación de la identidad relacionada hacia aquellas características comunes que conforman el deber ser nacional, también aparece su plural, “identidades”, a partir del establecimiento de una relación del sujeto con la interculturalidad y no con una cultura universal:

Décadas atrás, la escuela intervenía, desde un lugar privilegiado, en la conformación identitaria de los ciudadanos/as, intentando ocultar las diversidades culturales y las desigualdades socioeconómicas y fortaleciendo así la idea de una cultura hegemónica y homogeneizante. La identidad construida en función del rol de las personas dentro de la institución educativa (docente y alumno/a) dominaba por sobre las otras identidades que las conformaban. La heterogeneidad que siempre ha caracterizado a los sujetos estaba invisibilizada (DGCyE, 2007: 22)

Aunque la escuela está llamada a seguir formando al ciudadano (la identidad nacional), también es cierto que en nuestro presente se tiende a pensar que la ciudadanía, la pertenencia a un Estado-nación, no implica identidades homogéneas y que los ciudadanos de un Estado también pueden "pertenecer y construir sus identidades en virtud de otros aspectos ligados a la etnia, la religión, el género" (Grinberg y Levy, 2009: 136). El saber en el que se sostienen estas identidades es un acontecimiento en la superficie de procesos en los cuales disciplinas como la historia y la sociología han construido una persona determinada en un tiempo cronológico y en un espacio geográfico. ${ }^{3}$ Esto tiene como efecto un marcado multiculturalismo, que, en palabras de Zizek y Jameson, refiere a "esa actitud que -desde una suerte de posición global- trata a cada cultura local como el colonizador trata al pueblo colonizado: como nativos cuya mayoría debe ser estudiada y "respetada cuidadosamente", (Zizek y Jameson, 1998: 172).

El multiculturalismo produce un cambio de mirada en el efecto de la fragmentación, y un efecto de sentido que pone la diferencia individual como punto de partida para la formación de la subjetividad. En este haz de relaciones, se conforma un discurso sobre las competencias que involucra el reemplazo de un currículum enciclopédico, que prioriza contenidos disciplinares, por un modelo curricular flexible, interdisciplinario, centrado en el aprendizaje, entre otros rasgos o atributos distintivos. El desplazamiento de lo universal a la diferencia surge como una ruptura hacia los aprendizajes academicistas recurrentes que no tienen el valor de aportar capacitación alguna, ya que una vez aprendidos y evaluados se desvanecen. Los contenidos ahora son el medio para la adquisición de competencias aptas y funcionales para la sociedad y al mismo tiempo para desarrollar las potencialidades del hombre.

\section{DE LOS UNIVERSALES A LAS PROPIAS APTITUDES}

El nuevo haz de relaciones que conforman a la educación pone el énfasis en que lo experimentado en la práctica educativa "pueda ser empleado como recurso o capacitación adquirida en el desempeño de cualquier acción humana, no sólo en las de carácter manual, sino también en las conductuales, intelectuales, expresivas o de comunicación" (Cf.

Esto también ha implicado un cambio en otras disciplinas, como las ciencias sociales, donde se evidencia un retorno a un compuesto de personas describibles y casos analizables. 
Sacristán, 2008: 16). Así, las competencias constituyen una guía para el curriculum y un capital de inversión para el alumno.

Como establece el Diccionario de la Real Academia Española, competencia (del lat. competentīa; cf. competente), refiere a "incumbencia", "pericia, aptitud, idoneidad para hacer algo o intervenir en un asunto determinado" o "atribución legítima a un juez u otra autoridad para el conocimiento o resolución de un asunto". Incumbencia (de incumbir) refiere a "obligación y cargo de hacer algo"; y competente (del lat. compětens, -entis) se define como aquel "que tiene competencia", "que le corresponde hacer algo por su competencia". A su vez, una segunda acepción de competencia (del lat. competentǐa; cf. competir) refiere a "disputa o contienda entre dos o más personas sobre algo", "oposición o rivalidad entre dos o más que aspiran a obtener la misma cosa".

"Competencias" refiere, entonces, al desarrollo de capacidades y cualidades que son necesarias para competir en las sociedades modernas. Y, como hablar de competencias implica una práctica que desarrolle procedimientos y aptitudes para ser empleables en la vida, para mejorar la productividad, la escuela que esto propone se considera a sí misma funcional a la sociedad (Sacristán, 2008).

La mayor parte de los análisis sobre economía globalizada convergen en que el desafío esto [sic] presente, sólo se supera con el desarrollo de los recursos humanos, y esto depende básicamente de la educación. ¿Por qué? Porque es el valor agregado que concurre a la formación del capital humano [...] Si analizamos comparativamente el mundo y las políticas mantenidas en este sentido por los países desarrollados o aquéllos en vías de desarrollo pero que no han alcanzado importantes niveles en los últimos treinta años, veremos que los diferentes indicadores permiten verificar la necesaria correlación entre educación y desarrollo (DGCyE, 1998: 3).

Por ello, la educación es considerada como un "proceso mediante el cual se desarrollan las potencialidades del hombre, en tanto persona individual e integrante de una comunidad" (DGCyE, 2001: 10), y busca "propiciar que los alumnos pongan en juego sus diferentes competencias -cognitivas, perceptivas, lógico motrices, emocionales, relacionales, expresivas, coordinativas y condicionales- en la resolución de problemas motores y acrecienten su autonomía en lo que respecta a su propia constitución corporal" (DGCyE, 2010:19).

La competencia establece un saber que se evidencia en la acción, y en el cual no es el contenido el que guía la enseñanza, sino la resolución de problemas, "actividades que le permiten al sujeto convertirse en gestor de su propia existencia" (Grinberg y Levy, 2009: 111). Entonces, la educación tiene como finalidad hacer diligencias conducentes al logro de una tarea, despliega la capacidad de actuar, pasar de un saber declaratorio a un saber que se pueda implementar. Si el alumno puede implementar la habilidad, entonces tiene competencias.

En estos nuevos escenarios de emergencia, la formación del cuerpo ya no se basa en qué conocer acerca del cuerpo, la ciudadanía, la salud, sino en qué es capaz de crear con eso que se conoce y se sabe, y, esta competencia es sólo valorada en el mercado de trabajo donde se determina si el sujeto es apto para un empleo o no. Una educación por competencias, inmersa en la sociedad de la liberación, pone el acento en la gestión que "viene a aparecer como la forma de hacer frente a esta nueva realidad que, a la vez, se presenta como flexible y abierta en tanto brinda la posibilidades de realización personal" 
(Grinberg, 2006: 108). Ya no hay un plan de gestión a seguir que produzca determinada experiencia, ahora el individuo es el autor de su propio destino.

Acudimos a la configuración de una formación de saber-poder que, en el campo de la educación, en una primera etapa -años noventa- ha puesto el acento en la descentralización como eje del ejercicio democrático y participativo, en el que conceptos tales como "gestión", "proyecto", "innovación", "reflexión", "autonomía", "nueva escuela", "cambio", "participación/democracia", "autogestión", se afianzaron como el nuevo paisaje discursivo (Ball, 1994 y 1997; Da Silva, 1998; Popkewitz, 1996; Grinberg, 2008). Estas condiciones de posibilidad tienen un efecto de sentido en las reformas que se gestaron a partir del año 2004, que queda plasmado en objetivos educativos que apuestan a la búsqueda de la conciencia del cuerpo, y el Yo, en líneas generales, a la formación de la persona autónoma; característica que será tomada como una competencia del hombre.

En la propuesta realizada por la Unión Europea, se han descrito ocho competencias: competencia en comunicación lingüística, competencia matemática, competencia digital, competencia social y ciudadana, competencia cultural y artística, competencia en el conocimiento y la interacción con el mundo físico, tratamiento de la información y competencia digital, competencia para aprender a aprender, y autonomía e iniciativa personal.

Este nuevo dispositivo pedagógico, que incluye la autonomía personal como objetivo fundamental, se entreteje en una red de saberes que definen al sujeto a partir de la "esencia", "alma”, "yo", "conciencia”, de modo que al entrar en conexión con el exterior ensambla un ser autor de su propio destino. El dispositivo pedagógico se reconstruye en una práctica cuyo saber sobre el sujeto determina la posibilidad de pensarlo en las reglas de una autonomía, de una gestión de sí mismo, de su propio camino. En otras palabras, al sujeto como autónomo y gestor de sí mismo.

\section{EL MODO DE PENSAR EL SUJETO}

En una primera aproximación, si la Educación Secundaria coloca el individuo, su identidad, su contexto, su diferencia, como centro del proceso, ¿qué sentido tiene la educación? El desplazamiento que se produce en el curriculum lleva a poner en tensión al sujeto universal y el efecto homogeneizante de la educación, produciendo una apertura hacia el sujeto singular: el individuo. Esto coloca al sujeto como posible de ser fundado desde sí mismo y por sí mismo, pues queda como amo o iniciador de su discurso, sin relación con un exterior, con una estructura significante. $\mathrm{Al}$ no haber un único modelo válido de ser ciudadano, de ser saludable, la pregunta por quién soy se transforma en el objetivo.

Una red de elementos continuos y ciertas formaciones del discurso señalan, cercan y producen la formación de la diferencia y lo individual en pos de la autonomía, es decir, en pos del sujeto Amo de su discurso. Este modo de pensar la educación del cuerpo a partir de la constitución del sí mismo (yo-cuerpo), es una manera de efectuar una búsqueda retrospectiva hacia el fundamento subjetivo, suponiendo que nos constituimos en subjetividades por un interior plegado de fuerzas. Se supone que existe un individuo que a partir de su conciencia de sí mismo posee una certeza del mundo y del propio cuerpo. Esto coloca al alumno como centro del proceso y como guía de su propio aprendizaje, ya que el contenido de la educación queda subordinado por el individuo, quien será amo del 
discurso. En otras palabras, frente a este modo de pensar el sujeto, en el cual éste es creador de mundo, la tarea de la educación será "asistir a los alumnos para que logren actuar y tomar decisiones, convirtiéndose en expertos responsables de la gestión de sí mismos” (Grinberg y Levy, 2009: 114). Esto no quiere decir que la educación se despolitice; por el contrario, esta posición sobre el sujeto tiene una dimensión política en el sentido de constitución de lo humano, y también del curriculum como "configuración discursiva que anuda una red de sentidos que habilita la acción del sujeto" (Bordoli, 2013: 201). Por ejemplo, hace de la autonomía una competencia, que refiere "a la "condición de quien, para ciertas cosas, no depende de nadie" (RAE).

El problema que se plantea es que el sujeto sale de su individualidad, se descompone como propiedad en los límites de su propia imposibilidad de ser individual, autónomo, autocontenido. El sujeto no es causa de sí mismo, o de una conciencia de sí. "El lenguaje es causa del sujeto, entonces se pierde la posibilidad de que éste sea causa de sí mismo" (Eidelsztein 2012: 10). Para Lacan, de hecho, más que el lenguaje es el orden significante lo que se sitúa como causa del sujeto. Por ello, "ese sujeto es lo que el significante representa, y no podría representar nada sino para otro significante" (Lacan, 2010: 795). Si el sujeto se inscribe como representado entre al menos dos significantes, no puede darse una educación en referencia inmediata a un cogito, a una conciencia de sí, a un interior del cuerpo fundador. El sujeto del lenguaje no hace el discurso, sino "que está hecho por el discurso, e incluso está atrapado en él, es el sujeto de la enunciación” (Lacan, 2011: 53). De esta manera, cualquier idea de profundidad e interioridad se disuelve inmediatamente. El lenguaje captura al sujeto, no hay una conciencia, sino un inconsciente estructurado por el lenguaje, por el "discurso del Otro".

El sujeto definido desde una epistemología del lenguaje queda así despojado de un interior que se exteriorizaría, pues "no es poseedor de nada, ni de su lenguaje, ni de su conciencia, ni siquiera de su saber" (Foucault, 2013: 115). La historia del saber del cuerpo no ha quedado en el cuerpo, sino en el lenguaje, porque cuando se lo interroga no interviene una conciencia sino en el marco dentro del cual hablamos, el lazo discursivo. Esta teoría del sujeto nos permite identificar las reglas discursivas que fundamentan la Educación Física, y la apuesta a la búsqueda de la autonomía como objetivo a alcanzar. "La educación se presenta como espacio en el que estas lógicas se producen y reproducen; en el que los sujetos son llamados a hacerse y autohacerse así como la escuela que reinterpretada como institución de la comunidad queda en un vaivén sin hamaca librada a la gestión de sî" (Grinberg, 2006: 6).

El lenguaje es exterior, se encuentra ya hecho y ni siquiera puedo reemplazarlo por completo. El lenguaje es causa del sujeto, ya que "la causa no es, como se dice también del ser, un señuelo de las formas del discurso -se lo habría disipado ya. Perpetúa la razón que subordina al sujeto al efecto del significante" (Lacan, 2010: 798). Si el orden significante es la causa del sujeto, éste no es el fundador de sí mismo, ni hecho por la conciencia o por la materia biológica, sino que ha sido creado, "existe a partir de la nada" (Eidelsztein, 2012: 11). El problema que se plantea es que la experiencia originaria establece una primera complicidad con el mundo que permitiría nombrarlo, designarlo y finalmente conocerlo. Así, el sujeto sería el acto de fundación de sí mismo y del conocimiento del mundo, lo cual se rompe porque el sujeto nace por efecto del lenguaje. Por ello, "el hombre se convierte en humano cuando ingresa en el lenguaje e inaugura así la temporalidad de la historia" (Castro, 2008: 41). 
Estudios Pedagógicos XLII, Nº 1: 299-309, 2016

GESTIÓN DE SÍ Y EDUCACIÓN DEL CUERPO EN CONTEXTO: PERSPECTIVAS Y TENSIONES DE LA EDUCACIÓN ARGENTINA

La teoría de la enseñanza continúa partiendo del individuo como máquina biológica, psicológica, y ahora consciente, y coloca al sujeto en una "existencia anterior a su relación con el conocimiento y el lenguaje" (Behares, 2007: 4). Sin embargo, si el lenguaje es anterior al sujeto, no sólo no es posible pensar una enseñanza basada en una percepción originaria, sino tampoco como vivencia motriz. El lenguaje está implicado en el saber a enseñar y en relación con el sujeto. Por lo tanto, el sujeto queda unido en esa operación y se constituye como objeto. Al respecto Behares dice:

el hombre se constituye como tal por el acto de incorporación al lenguaje y no por ninguna otra cosa; el hombre se convierte en tal por su inscripción en el lenguaje. Esa inscripción en el lenguaje es indistinguible de una inscripción en el saber; estar en el lenguaje es estar en el saber y, por lo tanto, estar en posición de enseñanza (Behares, 2013: 247).

Esto no implica que el sujeto no pueda ser libre de pensamiento, innovador o revolucionario, sino que no podrá ser autor, más allá del saber, de las condiciones sociales, políticas, culturales que establecen las reglas del sentido de una época. "Ni siquiera los científicos pueden hacer descubrimientos o inventos más allá del contexto teórico de su momento" (Eidelsztein, 2012: 50). No hay una complicidad inicial con las cosas, sino que nuestras interpretaciones están construidas en un sistema de relaciones y condiciones formales.

\section{CONCLUSIONES}

El haz de relaciones sociales, históricas y políticas que construyen nuevos enfoques en el sistema educativo han establecido una individuación en la dialéctica individuo-sociedad, colocando al individuo como actor y autor de su propia identidad. Esta reconfiguración discursiva conforma un marco curricular en el que el contexto pasa a constituirse en un significante clave a la hora de pensar el acto educativo. En esta línea, el binomio escuela/ medio (contexto) se vio alterado, pues ya no "es el medio en términos universales que operaba como patrón para pensar el acto educativo; ahora el mismo es una categoría particular para pensar (...) el curriculum en contexto de pobreza y marginación" (Bordoli, 2013: 196). En efecto, la enseñanza se construye en relación a contextos particulares y de manera diferencial y por lo tanto los contenidos de la educación se particularizan: hay una educación para pobres y una educación para ricos, así como hay un deporte social o un deporte competitivo.

Este desplazamiento que coloca la diferencia, el sí mismo y el contexto, fragmenta los marcos normativos, la institución civilizadora, la formación de subjetividades en un determinado patrón cultural (Elías, 1987), el carácter conservador de la educación.

El dispositivo pedagógico se reconstruye en una práctica cuyo saber sobre el sujeto determina la posibilidad de pensarlo en las reglas de una autonomía, de una gestión de sí mismo, de su propio camino. Hay un saber del sujeto que lo coloca como natural, consciente e individual, y un conjunto de estrategias vinculadas con esa discursividad que produce y determina nuevas relaciones entre el sujeto y la educación, que no sólo refieren a discursos curriculares, sino también a cuestiones generales relativas a su recurrencia en nuestras sociedades: el sujeto como individuo y gestor de sí. 


\section{REFERENCIAS BIBLIOGRÁFICAS}

Agamben, G. (2009). Signatura rerum, Buenos Aires: Adriana Hidalgo Editora.

Behares, L. (2013). "Sobre las éticas de lo imposible y la enseñanza", en Southwell, M. y Romano, A. (comps.). La escuela y lo justo. Ensayos acerca de la medida de lo posible, Buenos Aires: UNIPE, Editorial universitaria, 245-260.

. (2007). "Enseñar en cuerpo y alma: la teoría de la enseñanza y el saber en la pulsión", en Educação Temática Digital, Campinas, Vol. 8, Nº esp., jun, 1-21.

(dir.) (2004). Didáctica mínima. Los acontecimientos del saber, Montevideo: Editorial Psicolibros, Waslala.

Bordoli, E. (2013). "Revisitando la enseñanza y lo curricular. Apuntes para pensar la igualdad", en Southwell, M. y Romano, A. (comps.). La escuela y lo justo. Ensayos acerca de la medida de lo posible, Buenos Aires: UNIPE, Editorial universitaria, 179-211.

Castro, E. (2008). Giorgio Agamben: una arqueología de la potencia, Ciudad Autónoma de Buenos Aires: UNSAM.

Dirección General de Cultura y Educación de la provincia de Buenos Aires [DGCyE] (2011). Diseño Curricular para la Educación Secundaria: $6^{\circ}$ año, Educación Física, La Plata. . (2010). Diseño Curricular para la Educación Secundaria: $4^{\circ}$ año, Educación Física, La Plata. . (2008). Diseño Curricular para la Educación Secundaria: $3^{\circ}$ año, La Plata. . (2007). Marco General de Política Curricular, La Plata. . (2006). Diseño Curricular para la Educación Secundaria: $1^{\circ}$ año ESB, La Plata. (2003). Aportes para la construcción curricular del área de educación Física, Serie de desarrollo curricular, Tomo I, La Plata. . (2001). Diseño Curricular. Marco General, La Plata. . (1998). Resolución $N^{\circ} 4625$ de Polimodal, 6 de agosto de 1998, La Plata.

Dussel, I. (2010). "El curriculum", en Explora. Las ciencias en el mundo contemporáneo, Programa de Capacitación Multimedial, Argentina, Ministerio de Educación.

Eidelsztein, A. (2012). "El origen del sujeto en psicoanálisis. Del Big Bang del lenguaje y el discurso en la causación del sujeto", en El Rey está desnudo. Revista para el psicoanálisis por venir, Año 4, N ${ }^{\circ}$, Buenos Aires: Letra Viva, 7-55.

Elias, N. (1987). El proceso de civilización, Madrid, Fondo de Cultura Económica.

Foucault, M. (2013). La inquietud por la verdad: Escritos sobre la sexualidad y el sujeto, Buenos Aires: Siglo XXI.

Grinberg, S. y Levy, E. (2009). Pedagogía, curriculum y subjetividad: entre pasado y futuro, Bernal: Universidad Nacional de Quilmes.

Grinberg, S. (2006). "Educación y gubernamentalidad en las sociedades de gerenciamiento", en Revista Argentina de Sociología, Año 4, $\mathrm{N}^{\circ}$ 6, 67-87.

Lacan, J. (2011). Mi enseñanza, Buenos Aires: Paidós. . (2010). "Posición del inconsciente", en Escritos 2, Buenos Aires: Siglo XXI.

Palamidessi, M. (2000). "Currículum y problematizaciones: moldes sobre lo cotidiano", en Gvirtz, S. Textos para repensar el día a día escolar. Sobre cuerpos, vestuarios, espacios, lenguajes, ritos y modos de convivencia en nuestra escuela, Buenos Aires: Santillana.

Sacristán, G. J. (Comp.). (2008). Educar por competencias, ¿qué hay de nuevo?, Madrid: Morata.

Tedesco, J. C. (1993). Educación y sociedad en la Argentina (1880-1945), Buenos Aires, Ediciones Solar.

Tenti Fanfani, E. (2002). "Voz socialización”, en Altamirano, C. (comp.). Términos críticos de la sociología de la cultura, Buenos Aires: Paidós.

Zizek, S. y Jameson, F. (1998). Estudios culturales. Reflexiones sobre el multiculturalismo, Buenos Aires: Paidós. 
